\title{
FINGER KNUCKLE-PRINT IDENTIFICATION BASED ON LOCAL AND GLOBAL FEATURE EXTRACTION USING SDOST
}

\author{
Mahesh Kumar, N.B. and K. Premalatha \\ Department of CSE, Bannari Amman Institute of Technology, Anna University, Tamilnadu, India
}

Received 2014-03-07; Received 2014-03-15; Accepted 2014-04-03

\begin{abstract}
Finger knuckle-print biometric system has widely used in modern e-world. The region of interest is needed as the key for the feature extraction in a good biometric system. The symmetric discrete orthonormal stockwell transform provides the computational efficiency and multi-scale information of wavelet transforms, while providing texture features in terms of Fourier frequencies. It outperforms leading wavelet-based texture analysis methods. This motivates us to propose a new local and global feature extractor. For the finger knuckle-print, the local and global features are critical for an image observation and recognition. For the finger knuckle-print, the local and global information are critical for an image observation and recognition. The local features of an enhanced finger knuckle-print image are extracted using symmetric discrete orthonormal stockwell transform. The Fourier transform of an image is obtained by increasing the scale of symmetric discrete orthonormal stockwell transform to infinity. The Fourier transform coefficients extracted from the finger knuckle-print image is considered as the global information. The local and global information are physically linked by means of the framework of time frequency analysis. The global feature is exploited to refine the arrangement of finger knuckle-print images in matching. The proposed scheme makes use of the local and global features to verify finger knuckle-print images. The weighted average of the local and global matching distances is taken as the final matching distance of two finger knuckle-print images. The investigational results indicate that the proposed work outperforms an existing works with an equal error rate of 0.0045 and $100 \%$ correct recognition rate on the finger knuckle-print database.
\end{abstract}

Keywords: Symmetric Discrete Orthonormal Stockwell Transform, Region of Interest, Fourier Transform, Local Feature, Global Feature

\section{INTRODUCTION}

In olden days, the metrics like keys, cards, passwords, PIN, are used for identity verification in computer systems. Sometimes these metrics may often be forgotten, revealed or altered. The biometric systems are designed for a reliable and accurate identification/verification. Biometrics can use physical characteristics like fingerprints (Ponnarasi and Rajaram, 2012), face, palmprints (Gayathri and Ramamoorthy, 2012; Hussein and Nordin, 2014), ear (Algabary et al., 2014), irises, or hand veins, or behavioural characteristics like speech, hand scripting or typing beat, to authenticate an individual. The personal traits are very difficult to lose or forget compared to the keys and passwords. Genetic traits are very difficult to copy. Hence biometric system is found to be safer and more secure than keys or passwords. The various applications of biometric technologies are in Forensic, Government, Commercial, Healthcare, Travel and Immigration. The biometrics like fingerprint, face, palmprint, iris, hand geometry has been studied broadly for human verification in several years. Multimodal biometric systems are also used to reduce the error rates and enhance the usability for personal identification has been presented by (Krishneswari and Arumugam, 2012; Corresponding Author: Mahesh Kumar, N.B., Department of CSE, Bannari Amman Institute of Technology, Anna University, Tamilnadu, India 
Mathivanan et al., 2012). Hand-based biometrics has been widely used in this e-world among the various biometric systems. The finger knuckle-print is extremely distinctive due to creases and skin folds and hence it is considered as a typical biometric verification system. It is not easily destroyed since people typically hold stuffs with an inside of the hand. It has a high user acceptance because it does not contain criminal investigation as in the fingerprint. Therefore, the finger knuckle-print feature has a large likely to be broadly identified as a biometric authentication system.

The local orientation information is extracted by Gabor filter and Fourier transform of an image is taken as global features obtained by rising the scale of the Gabor filter was conducted by (Zhang et al., 2011). (Kumar et al., 2013a) proposed the Fuzzy binary decision tree algorithm which is used for decision making on two classes: Genuine and imposter using matching scores computed from the biometric databases. Kumar et al. (2013b) projected an Ant colony optimization based fuzzy binary decision tree for bimodal finger knuckle-print verification system. The unique line and joint features in finger knuckleprint are not easily extracted by the subspace analysis schemes. The outer surfaces of finger joints in Finger Knuckle-Print (FKP) images have more line features than palm surface of the palm print image and finger knuckle-print have much lesser area than palm surface. A new biometric technique called as the finger knuckleprint is proposed in this study. Feature extraction and pattern matching of FKP images are focused mainly in this proposed work. The features in FKP images can be classified as local and global features based on the area of pixels involved in the feature extraction. The local information is a measure calculated within the local area and encoding the complete qualities within this area. Global information is a measure extracted from every pixel in the image. Hence the global information reflects a few holistic quality of the scrutinized image. Hence finger knuckle-print biometric system is classified into local based methods and global based methods proposed by (Muralidharan and Chandrasekar, 2012) based on both features.

The local-global feature fusion for finger knuckleprint system is used only in few papers. The local and global feature is important for an image observation. The recognition of human lives is believed in the field of psychophysics and neurophysiology. A global feature is appropriate for granular representation. The complete individualities of FKP images are reproduced in the global feature. The additional complete information within the particular region is encoded in the local feature. It is appropriate for better representation. The better recognition accuracy is obtained by fusing the local and global feature appropriately. Raut et al. (2014) proposed the Kekre's wavelet transform along with image enhancement is estimated on organized FKP database. A Riesz transforms is utilized to encode the local patterns of FKP images has been presented by (Zhang and $\mathrm{Li}, 2012$ ).

Baranidharan and Ghosh (2012) decribed the extraction of global features using Hilbert Transform and classifiation using Genetic Algorithm Optimized Elman (GAOE) Neural Network. Morales et al. (2011) suggested the Gabor filter technique to enhance FKP images and Scale Invariant Feature Transform (SIFT) is used to extract the features. Although the previous works demonstrated that the finger knuckle-print are unique, however they have some disadvantages that mainly uses 3D shape features of FKP images rather than texture images and subspace analysis method's are not successfully extract the distinctive features in FKP images. Zhang et al. (2011) used the Gabor feature and Fourier coefficients as local and global information in FKP images. A new Weighted Competitive coding (WCompCode) scheme for effective feature matching was propsoed by (Gao and Yang, 2013).

The remainder of this study is organized as follows. Section 2 introduces the materials and methods. Section 3 discused the results and section 4 depict the discussions. Section 5 reports the conclusion.

\section{MATERIALS AND METHODS}

\subsection{Overview of ST, DST, DOST, SDOST}

\subsubsection{Stockwell Transform}

The continuous Stockwell Transform (ST) of a signal $h$ $(t))$ is defined as Equation 1:

$$
s(\tau, f)=\int_{-\infty}^{\infty} h(t) \frac{|f|}{\sqrt{2 \pi}} e^{-(\tau-t)^{2} f^{2} / 2} e^{-i 2 \pi f t} d t
$$

where, $\mathrm{f}$ is the frequency variable, $\mathrm{t}$ is the time variable and $\tau$ is the time translation. The width of the Gaussian window function is proportional to the inverse of the frequency. The Stockwell spectrum $\mathrm{s}(\tau \mathrm{f})$ describes how frequency components in the signal differ over time. The Fourier spectrum of the signal is obtained if the Stockwell transform goes to infinity and is given by Equation 2:

$$
H(f)=\int_{-\infty}^{\infty} s(\tau, f) d \tau
$$


The inverse Stockwell transform is given by Equation 3:

$s(\tau, f)=\int_{-\infty}^{\infty} H(\xi+f) e^{-\frac{(2 \pi \xi)^{2}}{2 f^{2}}} e^{i 2 \pi \xi \tau} d f$

Also the ST is represented in the Fourier domain as Equation 4:

$s(\tau, f)=\int_{-\infty}^{\infty} H(\xi+f) e^{-\frac{(2 \pi \xi)^{2}}{2 f^{2}}} e^{i 2 \pi \xi \tau} d \xi$

where, $\mathrm{H}(\mathrm{f})$ is the Fourier spectrum of $\mathrm{h}(\mathrm{t})$ :

\subsubsection{Discrete Stockwell Transform}

Let $\mathrm{h}[\mathrm{l}]=\mathrm{h}(1, \mathrm{~T}), 1=0,1, \ldots \mathrm{N}-1$ be the samples of the continuous signal $\mathrm{h}(\mathrm{t})$, where $\mathrm{T}$ is the sampling interval. Its discrete Fourier transform is given by Equation 5:

$$
H[m]=\sum_{l=0}^{N-1} h[l] e^{-i 2 \pi m l / N}
$$

where, the discrete frequency index $\mathrm{m}=0,1, \ldots, \mathrm{N}-1$.

The Discrete ST(DST) is given by Equation 6:

$$
s[k, n]=\sum_{m=0}^{N-1} e^{-\frac{2 \pi^{2} m^{2}}{n^{2}} H[m+n] e^{\frac{i 2 \pi m k}{N}}}
$$

where, $\mathrm{K}$ is the index for time translation and $\mathrm{n}$ is the index for frequency shift. Function $\exp \left(-2 \pi^{2} \mathrm{~m}^{2} / \mathrm{n}^{2}\right)$ is Gaussian window in the frequency domain.

The inverse DST is given by Equation 7:

$$
h[l]=\frac{1}{N} \sum_{n=0}^{N-1}\left\{\frac{1}{N} \sum_{n=0}^{N-1} S[k, n]\right\} e^{i 2 n l / N}
$$

For a signal of length $\mathrm{N}$, the DST produces $\mathrm{N}^{2}$ number of coefficients in the time-frequency domain.

\subsubsection{Discrete Orthonormal ST}

An orthogonal time-frequency version of the Stransform is mostly used due to several reasons. An orthogonal transformation in DOST takes N-point time series to an $\mathrm{N}$-point time frequency representation. Hence the maximum competence of representation is attained. The transformation matrix is consider as the time series to the DOST representation. The orthogonal means that the inverse matrix is equal to the complex conjugate transpose. Due to orthogonal transformation, the vector norm is preserved in DOST. The norm of the time series equals the norm of DOST according to the Parseval theorem. An wellorganized depiction of the S-transform is obtained by applying the inner products between a time series $\mathrm{h}[\mathrm{kT}]$ and the basis functions. The parameters are frequency variable revealing of the centre of a frequency band $\mathrm{v}$ and similar to the voice of the wavelet transform, $\beta$ representing the width of the frequency band and $\tau$ a time variable Equation 8:

$$
S\{h[k T]\}=S\left(\tau T, \frac{v}{N T}\right)=\sum_{k=0}^{N-1} h[k T]\left(S_{[\theta, \beta, \pi]}[k T]\right)
$$

These basis functions $S[v, \beta, \tau][\mathrm{kT}]$ for the universal instance are given as Equation 9:

$$
S_{[\theta, \beta, \pi]}[k T]=\frac{i e^{-i \pi}}{\sqrt{\beta}} \frac{\left\{i e^{-i 2 \pi\left(k / N^{-\tau} / \beta\right)(v-\beta / 2-1 / 2)}\right\}}{2 \sin \left[\pi\left(k / N^{-\tau / \beta}\right)\right]}
$$

The sampling of the time-frequency space is not established at this point. To ensure orthogonality, various rules are applied to the sampling of the time-frequency space. Therefore the rules are:

- $\quad$ Rule 1. $\tau=0,1 \ldots \beta-1$

- Rule 2. $v$ and $\beta$ is used once and only once and it is selected for each Fourier frequency sample

Normally, the phase correction of the S-transform is different from the wavelet transform or the Gabor filter bank. Therefore, the parameters $\nu, \beta, \tau$ are integers welldefined and these functions formed as the basis. It contains one or more local time samples $(\tau)$ for each voice and the $\tau$ is equal to $\beta$. Hence the wider the frequency resolution (large $\beta$ ) and the more samples in time (large $\tau$ ) are obtained. It is considered as a significance of the uncertainty principle. The orthogonal basis functions have no vanishing moments compared to the wavelet function. The basis functions are not selfsimilar and also it is not translations of a single function.

\subsubsection{Symmetric DOST}

Unlike the FFT, DOST coefficients of a real-valued input signal are not conjugate symmetric. Therefore it is necessary to calculate the positive frequency coefficients and the negative part as well in real applications. DOST is slightly modified to achieve the conjugate symmetry but still maintaining its orthogonality. Hence this modified DOST is refereed as the Symmetric DOST (SDOST). For real-valued signals, half of the computation can be avoided if the symmetric DOST is used.

The basis functions for SDOST are obtained by imposing the conjugate symmetry requirement on the DOST coefficients and is given by Equation 10: 
$\mathrm{S}_{[\theta, \beta, \pi]}=\left(\mathrm{S}_{[-\theta, \beta, \pi]}\right)^{*}$

where, $a=p\left(\frac{k}{N}-\frac{t}{b}\right)$ is the center of the temporal window.

The orthonormal property for the basis vector of the symmetric DOST is verified due its definition. Due to the advantage of the FFT, DOST coefficients is calculated faster than by direct calculation (normally ten times faster). SDOST can be calculated in a similar way by multiplying the signal by a phase ramp. Note that the system matrix is slightly different for SDOST than for DOST. However, this transformation matrix is blockdiagonal in both cases. SDOST corresponds to a $1 / 2$ sample shift along the frequency axis due to the shift implemented using the Fourier shift theorem, there is no loss of information to re sampling.

\subsection{Preprocessing and ROI Extraction}

FKP images accumulated from dissimilar fingers and are very diverse. The spatial locations of the finger knuckle-print are dissimilar for each FKP images and hence it is aligned by constructing a local coordinate system for every FKP images. The local coordinate system determination and ROI sub-image extraction technique is presented by (Zhang et al., 2011). A Local Global Information Fusion (LGIF) scheme for finger knuckle-print recognition was proposed in this study. DOST is used to extract the instantaneous phase difference as the local feature because it is already used in palmprint verification system. The huge quantity of global features was attained by aggregating the scale of SDOST and hence the representation of an image local feature got weakened rapidly. SDOST is reduced to the Fourier transform of the complete image when its scale is increased to infinity. Hence, no local features are extracted but the good resolution for the global features of FKP image can be obtained. Therefore, Fourier transform coefficients are obviously measured as the global features in this study. The arrangement between intra-class FKP ROIs was refined by using global Fourier information. The two matching distances are calculated by comparing the local features and the global features at the pattern matching stage. Finally, the two matching distances are combined to get the final matching distance based on the fusion rule. Figure 1a shows the FKP image sensor device and Fig. 1b shows a sample finger knuckle-print image. Figure 1c and d shows ROI extraction technique explained by (Zhang et al., 2011).

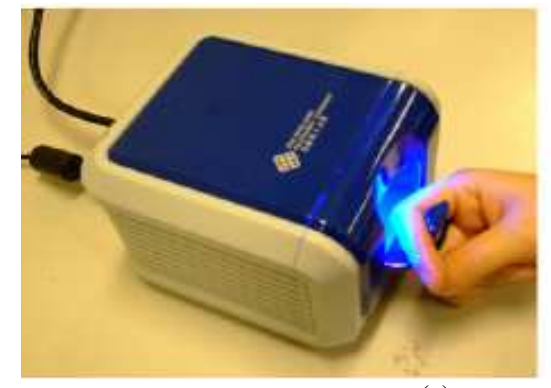

(a)

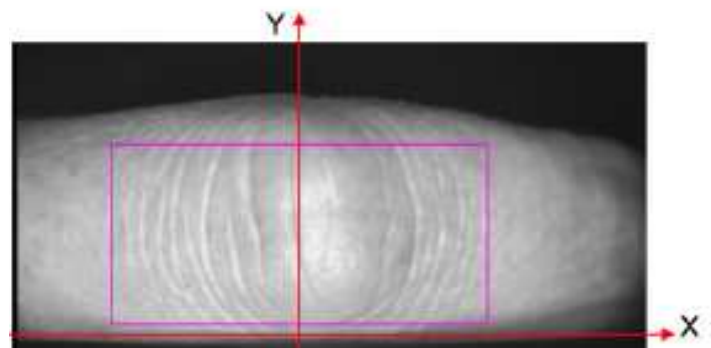

(c)

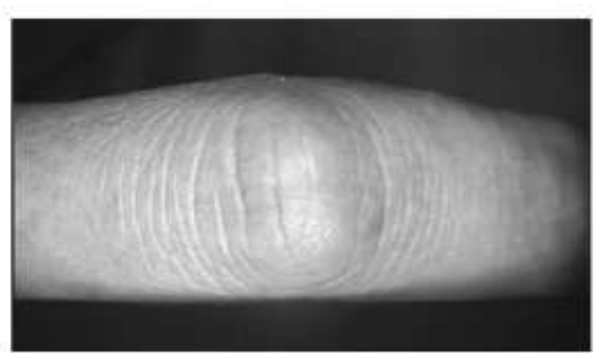

(b)

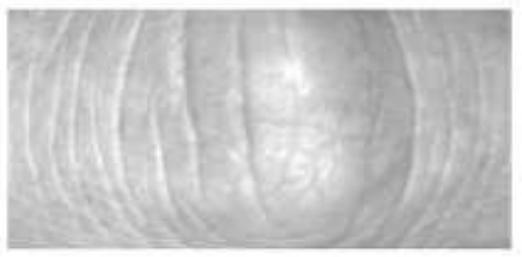

(d)

Fig. 1. (a) Image sensor device (b) Sample FKP image (c) ROI calculation and (d) ROI image 


\subsection{Local-Global Feature Extraction and Matching}

\subsubsection{Local Feature}

The texture features of finger knuckle-print are extracted by using 2D-SDOST after the completion of pre-processing and ROI segmentation. SDOST is used to extract the three basic textures like magnitude, phase and orientation. The most tough and unique local feature for Finger Knuckle print recognition is the local orientation information. The local orientation is taken as the local feature. Therefore orientation coding based feature extraction method is appropriate for images containing abundant line-like structures. It has the advantage of high precision, toughness to illumination variation and speed matching. When matching two FKP images, the angular distance based on Euclidean Distance is used.

\subsubsection{Global Feature}

SDOST is used to extract the local orientation information and also it can be considered as a windowed Fourier transform. The phase only correlation and band-limited phase only correlation are used to match the global feature of two FKP images described in this section.

\subsubsection{Phase-Only Correlation (POC)}

The Fourier transform coefficients extracted from SDOST are taken as the global information. Phase-only correlation method is used to match the two Fourier transforms of FKP image. In image registration tasks, POC based schemes are broadly used. POC has also been used as a resemblance measure in various biometrics systems. The Band-Limited Phase-Only Correlation (BLPOC) is more efficient than the typical POC method. The translation parameters between two FKP images in the Fourier domain 'are effectively evaluated by POC schemes. The translation property of the Fourier transforms is the fundamental principle of POC. The most significant property of POC match up to the ordinary correlation is its precision in image matching. Figure 2 Shows an example of image matching using POC function. When two images are same, their POC function gives a different severe peak. The peak falls considerably in POC function if two images are not same. Thus, POC function shows much greater discrimination ability than the normal correlation function. For pattern matching, the height of the peak is used as a resemblance measure.

\subsubsection{Band-Limited Phase-Only Correlation (BLPOC)}

The low and high frequency components are included in POC-based pattern matching schemes. But the high frequency components contain more noise. The band-limited POC (BLPOC) is used to remove noisy high frequency components. The range of the spectrum in the finger knuckle-print image is restricted in BLPOC. The ranges of the natural frequency band of FKP images are given by $\mathrm{u}=-\mathrm{U}_{0} \quad \mathrm{U}_{0}$ and $\mathrm{v}=-\mathrm{V}_{0, \ldots,}, \mathrm{V}_{0}$ where, $0<\mathrm{U}_{0} \leq \mathrm{M}_{0}, 0 \leq \mathrm{V}_{0} \leq \mathrm{N}_{0}$, Therefore the efficient size of spectrum is given by $\mathrm{L}_{1}=2 \mathrm{U}_{0}+1$ and $\mathrm{L}_{2}=2 \mathrm{~V}_{0}+1$ BLPOC function is given by Equation 11:

$\mathrm{p}_{\mathrm{gf}}^{\mathrm{U}_{0} \mathrm{v}_{0}}(\mathrm{~m}, \mathrm{n})=\frac{1}{\mathrm{~L}_{1} \mathrm{~L}_{2}} \sum_{\mathrm{u}=-\mathrm{U}_{0}}^{\mathrm{U}_{0}} \sum_{\mathrm{v}=-\mathrm{v}_{0}}^{\mathrm{U}_{0}} \mathrm{R}_{\mathrm{GF}(\mathrm{u}, \mathrm{v}) \mathrm{e}} \mathrm{j} 2 \pi\left(\mathrm{mu} / \mathrm{L}_{1}+\mathrm{nv} / \mathrm{L}_{2}\right)$

where, $\mathrm{m}=-\mathrm{U}_{0} \quad \mathrm{U}_{0}$ and $\mathrm{n}=-\mathrm{V}_{0, \ldots,}, \mathrm{V}_{0}$, BLPOC function also uses the properties of POC function. BLPOC function will have a different sharp peak when two images are like. Also the translational displacement between two images is estimated by the location of the peak. Experiments point out that BLPOC function provides a much higher discrimination capability than the original POC function in finger knuckle-print recognition. These examples indicate in the instance of genuine matching (a matching achieved between a pair of FKP images from the same hand), BLPOC will show a much sharper peak than POC for an imposter matching (a matching achieved between a couple of FKP images from dissimilar hand), neither BLPOC nor POC will display a different severe peak. Therefore, BLPOC is used to align the displacement between FKP ROI images. Finally the resemblance between Fourier transforms of the aligned ROIs is measured. Figure 3 shows BLPOC function.

\subsection{Local Global Information Fusion for FKP Recognition}

The Local Global Information Fusion (LGIF) based finger knuckle-print recognition algorithm is presented in this section. The finger knuckle-print image sensor device and ROI extraction method can reduce the geometric transformations between intra-class ROIs. But it is still unavoidable because there is some displacement between intra-class ROIs in the finger knuckle-print images. This will weaken the genuine matching scores. The minimum resulting matching distances was measured to be the final matching distance by converting one set of features in horizontal and vertical directions several times. 


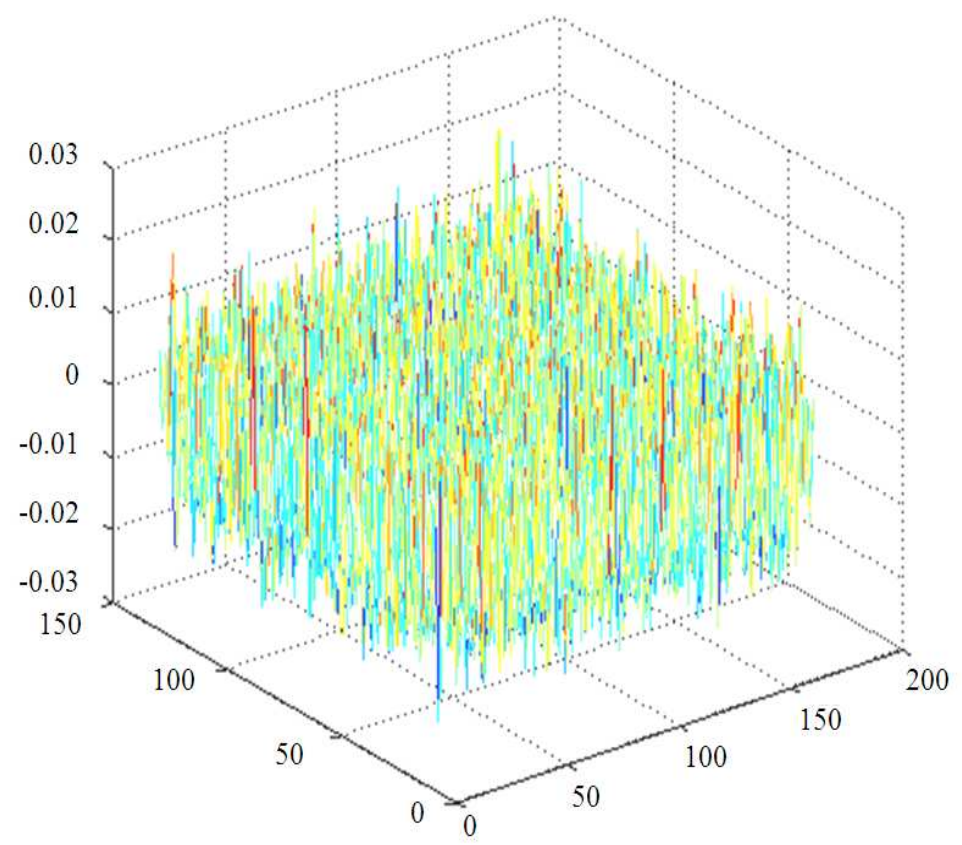

Fig. 2. POC function

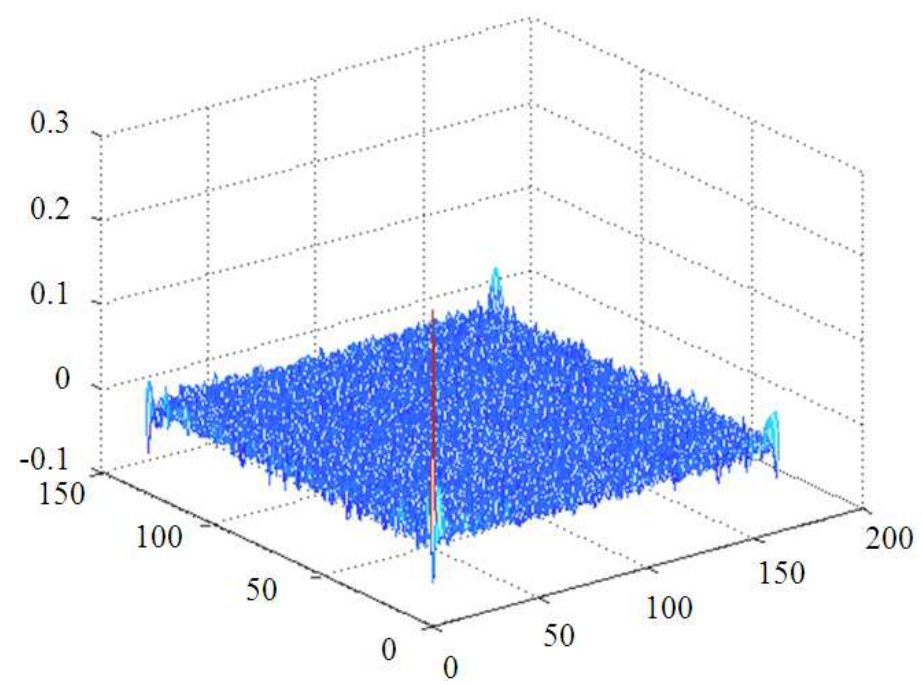

Fig. 3. BLPOC function

This problem can be solved in a different way by evaluating the translation parameters between two ROIs using BLPOC function. Then the common regions can be cropped based on which the feature matching is performed. The translation parameters between two finger knuckle-print ROI images can be estimated from the peak location of the global BLPOC of them. Then two FKP ROI images can be aligned based on the translation parameters and extracts the common regions. It should be noted that in the finger knuckle-print system, the ratio between the common region area and the area of the original ROI can be checked. Then, by matching two 
finger knuckle-print ROI images and the matching distance is obtained. The peak value of BLPOC function between finger knuckle-print ROI images is used to measure the similarity of their Fourier transforms. Then, the matching distance is obtained. Until now, two matching distances have been obtained. The final matching distance is obtained by combining the two distances. The two different matchers like local feature based matcher and global feature based matcher is used to get the two matching distances. The matching distances are fused based on the weights assigned according to an Equal Error Rate (EER) by using Maximum Weighted rule (MW). The weights are inversely proportional to the resultant EERs obtained on a training dataset by different matchers. Then, the fused final matching distance is calculated.

\section{EXPERIMENTAL RESULTS}

The authors strong-willed to use the PolyUFKP database consists of 7920 images collected from 660 different fingers. The samples are collected in 2 separate sessions. In each session; six images are collected for the left index and middle finger, the right index and middle finger. From each person, 48 images are collected from 4 fingers. The size of the acquired FKP images is $768 \times 576$ under resolution above 400 dpi. Based on the experiments, high resolution images are not necessary for feature extraction and pattern matching. Therefore, Gaussian smoothing operation is applied to the original image. The smoothed image is down sampled to about 150 dpi. Hence the size of ROI images is $110 \times 220$ pixels. Performance of the propsed system is calculated using the selected FKP ROI images extracted from the hand images of the individual databases. 5 images per finger knuckle-print are taken for training and 2 images are used for testing. Verification is a process in which a person can be uniquely identified by evaluating one or more distinguishing genetic traits. Performance of the propsed system has been analyzed with the help of four metrics like EER, Detection Error Trade-off (DET), Correct Recognition Rate (CRR) and Receiver Operating Characteristics (ROC) curves. The CRR of FKP recognition system is given by Equation 12:

$$
C R R=\frac{R 1}{R 2} \times 100
$$

Where:

$\mathrm{R}_{1}=$ Denotes the number of correct FKP images

\section{$\mathrm{R}_{2}=$ The total number of FKP images}

DET curve is obtained by adjusting the matching threshold. DET is defined as a plot of False Reject Rate (FRR) against False Accept Rate (FAR). DET curve is created for all possible thresholds. DET curves gives the general verification correctness of a biometric system. Figure 4 shows DET curve generated by the finger knuckle-print verification scheme. ROC curve is used to analyse the performance of FKP authentication system. ROC is a plot of Genuine Acceptance Rate (GAR) against False Acceptance Rate (FRR) shown in Fig. 5 The False Acceptance Rate (FAR) and False Rejection Rate (FRR) of a biometric system can be obtained by adjusting various thresholds. Therefore both FAR and FRR are inversely related. Thus, at a particular threshold, these two curves intersect. This intersect point is termed as an equal error rate. The EER is defined at the false accept rate is equal to the false reject rate.

EER is used to evaluate the verification accurateness. The Fourier transform of the complete image is kept as the feature in BLPOC. Hence the extraction of Fourier transform of the FKP image is called as a global-based method. Therefore, the investigational results show that the combining local and global features together performs better than the methods in the previous works depending on the local and global features. The Decidability Index (DI) is the measure of the separability of genuine and imposter matching scores. DI is given by Equation 13:

$$
D I=\frac{\left|\mu_{1}-\mu_{2}\right|}{\sqrt{\left(\sigma_{1}-\sigma_{2}\right) / 2}}
$$

The distance distributions of genuine matching's and imposter matching' acquired by proposed scheme are plotted as shown in Fig. 6. Performance comparison of different finger knuckle-print verification schemes is shown in Table 1.

Table 1. Performance comparison of different finger knuckleprint verification schemes

\begin{tabular}{lll}
\hline Methodology & EER (\%) & DI \\
\hline SIFT and SURF Matching & & \\
Scores (Badrinath et al., 2011) & 0.2150 & - \\
LGIC (Zhang et al., 2011) & 0.4020 & 4.5356 \\
Propsed Method - LGIF & 0.0045 & 5.1600 \\
\hline
\end{tabular}


Mahesh Kumar, N.B. and K. Premalatha. / American Journal of Applied Sciences 11 (6): 929-936, 2014

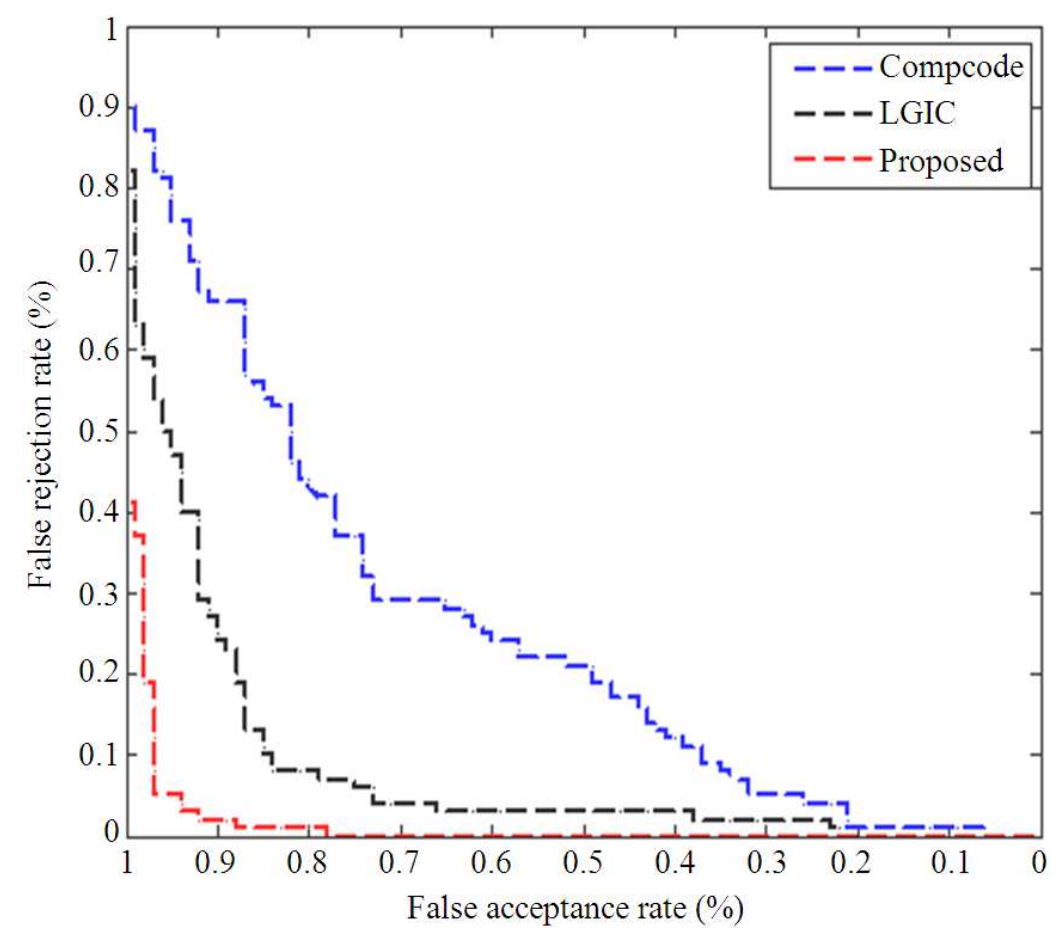

Fig. 4. DET curve

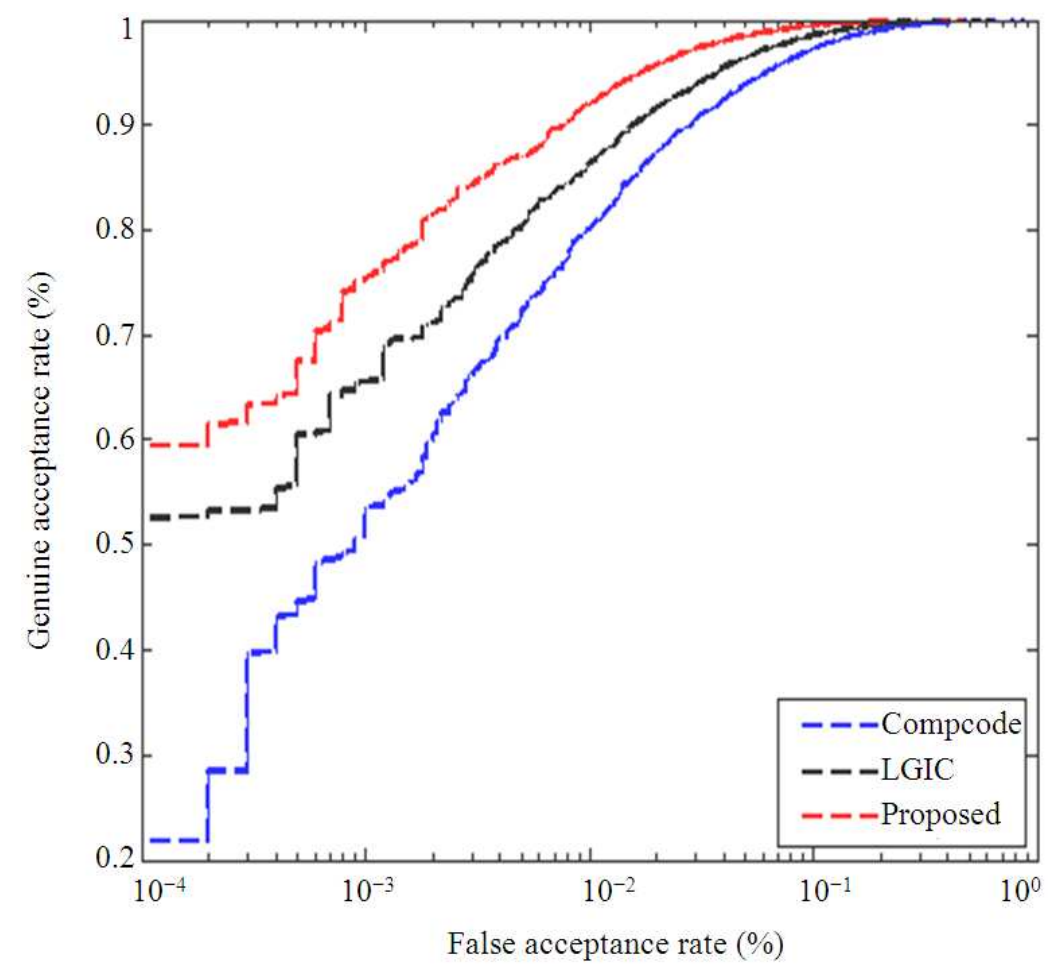

Fig. 5. ROC Curve 


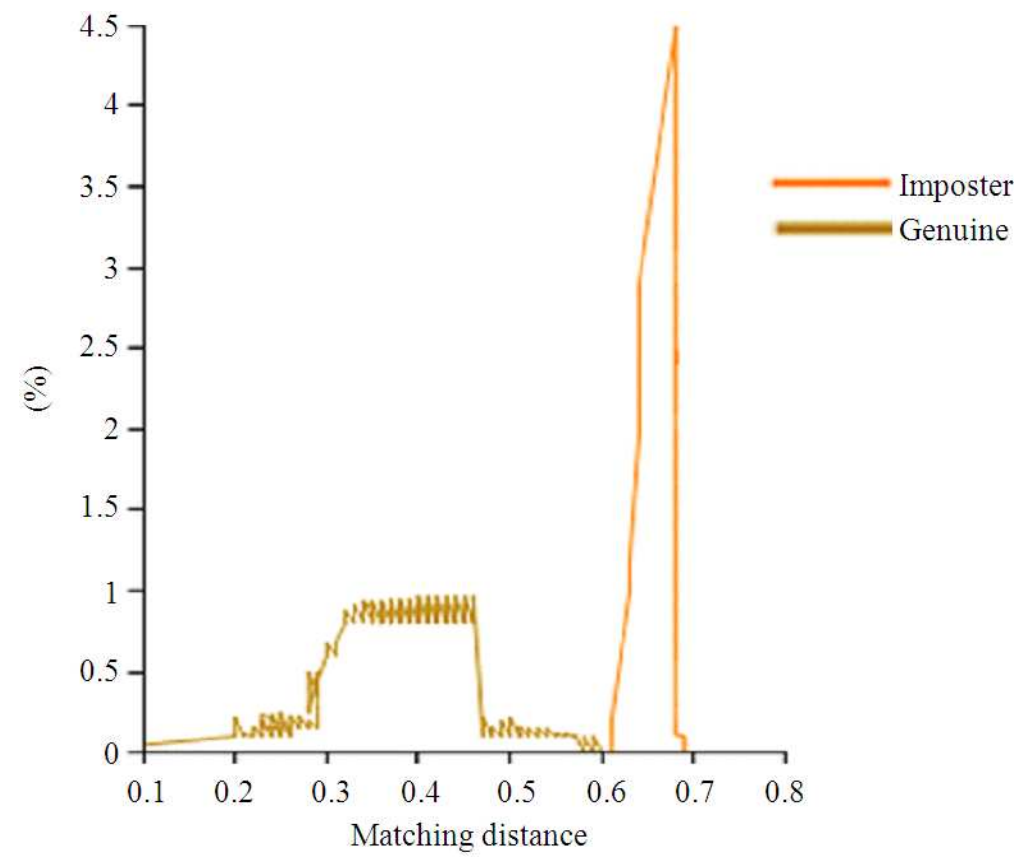

Fig. 6. Distance distributions of genuine matching's and imposter matching's

\section{DISCUSSION}

Finger knuckle print recognition system is an emerging biometrics system in modern e-world. As explained in the section of Introduction, Zhang et al. (2011) did some salient work to combine the local and global features in their system using Gabor filter to achieve EER of 0.402 and the proposed system could achieve an EER of 0.0045 by using SDOST. There are some variations occur in the same fingers collected at different sessions. Hence it results in severe affine transformations among intra-class FKP images. In future work, the effects of affine transformations can be reduced.

\section{CONCLUSION}

The local-global information fusion based finger knuckle-print biometric system was proposed in this study. Both local and global features are critical for an image observation. Therefore they play dissimilar and corresponding roles in this technique. The local orientation taken by SDOST was measured as the local feature. The Fourier transform can be obtained if the scale of SDOST is increased to infinity due to the fact of time-frequency analysis. Hence the Fourier transform of FKP images was obviously considered as the global feature in this proposed scheme. The arrangement of finger knuckle-print images in pattern matching is done by using the global features. The investigational results performed on FKP database show that the proposed method provides improved performance in terms of ERR and $100 \%$ CRR. In the future, the affine transformations between FKP images from the same finger are reduced to further improve the proposed system.

\section{REFERENCES}

Algabary, K.M.S., K. Omar and M.J. Nordin, 2014. 3Dimensional ear recognition based iterative closest point with stochastic clustering matching. J. Comput. Sci., 10: 477-483. DOI: 10.3844/jcssp.2014.477.483

Baranidharan, T. and D.K. Ghosh, 2012. Medical image classification using genetic optimized elman network. Am. J. Applied Sci., 9: 123-126. DOI: 10.3844/ajassp.2012.123.126

Badrinath G.S., A. Nigam and P. Gupta, 2011. An efficient finger-knuckle-print based recognition system fusing sift and surf matching scores. Proceedings of the 13th International Conference on Information and Communications Security, Nov. 2326, Springer-Verlag Berlin, Beijing, China, pp: 374387. DOI: 10.1007/978-3-642-25243-3_30 
Gayathri, R. and P. Ramamoorthy, 2012. Automatic personal identification using feature similarity index matching. Am. J. Applied Sci., 9: 678-685. DOI: $10.3844 /$ ajassp.2012.678.685

Gao, G. and J. Yang, 2013. Weight competitive coding for finger-knuckle-print verification. Proceedings of the 8th International Chinese Conference on Biometric Recognition, Nov. 16-17, Jinan, China, pp: 185-192: DOI: 10.1007/978-3-319-02961-0_23

Hussein, I.S.H. and M.J. Nordin, 2014. Palmprint verification using invariant moments based on wavelet transform. J. Comput. Sci., 10: 1389-1396. DOI: $10.3844 /$ jcssp.2014.1389.1396

Krishneswari, K. and S. Arumugam, 2012. Multimodal biometrics using feature fusion. J. Comput. Sci., 8: 431-435. DOI: 10.3844/jcssp.2012.431.435

Kumar, A., M. Hanmandlu and H.M. Gupta, 2013a. Fuzzy binary decision tree for biometric based personal authentication. Neurocomputing, 99: 87-97. DOI: 10.1016/j.neucom.2012.06.016

Kumar, A., M. Hanmandlu and H.M. Gupta. 2013b. Ant colony optimization based fuzzy binary decision tree for bimodal hand knuckle verification system. Expert Sys. Applic., 40: 439-449. DOI: 10.1016/j.eswa.2012.07.042

Mathivanan, B., V. Palanisamy and S. Selvarajan, 2012. A hybrid model for human recognition system using hand dorsum geometry and finger-knuckle-print. J. Comput. Sci., 8: 1814-1821. DOI: 10.3844/jcssp.2012.1814.1821
Muralidharan, R. and C. Chandrasekar, 2012. 3D object recognition using multiclass support vector machine-k-nearest neighbor supported by local and global feature. J. Comput. Sci., 8: 1380-1388. DOI: 10.3844/jcssp.2012.1380.1388

Morales, A., C.M. Travieso, M.A. Ferrer and J.B. Alonso, 2011. Improved finger-knuckle-print authentication based on orientation enhancement. Elect. Lett., 47: 380-381. DOI: 10.1049/el.2011.0156

Ponnarasi, S.S. and M. Rajaram, 2012. Impact of algorithms for the extraction of minutiae points in fingerprint biometrics. J. Comput. Sci., 8: $1467-$ 1472. DOI: $10.3844 /$ jcssp.2012.1467.1472

Raut, R.D., S. Kulkarni and N.N. Gharat, 2014. Biometric authentication using kekre's wavelet transform. Proceedings of the International Conference on Electronic Systems, Signal Processing and Computing, Jan. 9-11, IEEE Xplorer Press, Nagpur, India, pp: 99-104. DOI: 10.1109/ICESC.2014.22

Zhang, L., L. Zhang, D. Zhang and H. Zhu, 2011. Ensemble of local and global information for finger knuckle-print recognition. Patt. Recogn., 44: 19901998. DOI: 10.1016/j.patcog.2010.06.007

Zhang, L. and H. Li, 2012. Encoding local image patterns using Riesz transforms: With applications to palmprint and finger-knuckle-print recognition. Image Vis. Comput., 30: 1043-1051. DOI: 10.1016/j.imavis.2012.09.003 